\title{
Effect of strain and variable mass on the formation of antibonding hole ground states in InAs quantum dot molecules
}

\author{
J. Planelles, ${ }^{1, *}$ J. I. Climente, ${ }^{1}$ F. Rajadell, ${ }^{1}$ M. F. Doty, ${ }^{2}$ A S. Bracker,${ }^{3}$ and D. Gammon ${ }^{3}$ \\ ${ }^{1}$ Departament de Química Física i Analítica, Universitat Jaume I, E-12080 Castelló, Spain \\ ${ }^{2}$ Department of Materials Science and Engineering, University of Delaware, Newark, Delaware 19716, USA \\ ${ }^{3}$ Naval Research Laboratory, Washington, DC 20375, USA \\ (Received 15 July 2010; revised manuscript received 8 September 2010; published 4 October 2010)
}

\begin{abstract}
Using four-band $k \cdot p$ Hamiltonians, we study how biaxial strain and position-dependent effective masses influence hole tunneling in vertically coupled InAs/GaAs quantum dots. Strain reduces the tunneling and hence the critical interdot distance required for the ground state to change from bonding to antibonding. The reduced spin-orbit interaction in the GaAs matrix, which we account for using position-dependent Luttinger parameters, has the opposite effect. This compensation results in the critical distance being little affected. The possibility to induce the bonding-to-antibonding transition using longitudinal magnetic fields is also investigated. LuttingerKohn Hamiltonian predicts a magnetic enhancement of the heavy hole-light hole coupling which, in turn, leads to such transition. No such effect is, however, observed in magnetophotoluminescence experiments. An alternative implementation of the magnetic field in the envelope function Hamiltonian is given which retrieves the experimental behavior.
\end{abstract}

DOI: 10.1103/PhysRevB.82.155307

PACS number(s): 73.21.La, 73.40.Gk, 71.70.Fk

\section{INTRODUCTION}

More than a decade since they were first synthesized, artificial molecules made of coupled semiconductor quantum dots (CQDs) are still under intense research. ${ }^{1,2}$ This is largely due to their widely tunable electronic structure. The tunnel coupling between a pair of QDs can be modulated by means of structural design ${ }^{3}$ or after growth using external fields. ${ }^{1,2,4-6}$ The possibility to vary the strength of the molecular bond at will is not only useful for the development of applications in different fields, such as quantum information technology, ${ }^{3,7-9}$ quantum optics, ${ }^{10}$ spintronics, ${ }^{11}$ or light harvesting, ${ }^{12}$ but it has also unveiled new physics which had never been observed in natural molecules.

A most prominent example is the recent discovery of a molecular ground state of antibonding character in $p$-charged InAs/GaAs CQDs. ${ }^{13}$ This kind of state had been anticipated by atomistic calculations. ${ }^{14-17}$ An understanding of its origin and the conditions for it to occur were provided by some of us using multiband $k \cdot p$ theory. ${ }^{18}$ Unlike in single QDs, where valence holes are well described as heavy holes (HHs), mixing with light holes (LHs) becomes relevant in vertically CQDs. The simplest description of holes accounting for this mixing is the four-band Luttinger-Kohn (LK) Hamiltonian, which includes mixing of $\mathrm{HH}$ and $\mathrm{LH}$ subbands. ${ }^{19}$ Within this model one can show that the tunneling rate of holes is mainly given by the tunneling of $\mathrm{HH}$ orbitals. However, there is an additional correction induced by the HH-LH interaction which opposes the $\mathrm{HH}$ tunneling. When the QDs are closely spaced, the correction is negligible. However, when the interdot distance increases and $\mathrm{HH}$ tunneling becomes small, the correction starts dominating. At this point the net tunneling rate becomes negative and the molecular ground state is essentially an antibonding wave function. ${ }^{13,18}$

The realization of antibonding ground states suggests opportunities to engineer new optoelectronic devices, and it has motivated a number of subsequent studies. Hsieh et al. ${ }^{20}$ investigated the implications for hole spin-based qubits. Implications for charged based qubits were also explored. ${ }^{21}$ Chwiej and Szafran ${ }^{22}$ showed that the split-off band had a minor influence on the formation of antibonding ground states. Climente predicted that the bonding-to-antibonding ground-state transition could be induced reversibly using magnetic fields. ${ }^{23}$ Yakimov et al. ${ }^{24}$ showed that a similar behavior can be expected in diamond structure semiconductors, namely, Ge/Si CQDs. An antibonding hole ground state has been also detected very recently in GaAs/AlGaAs CQDs. ${ }^{25}$

Despite this progress, an important question remains open. To date, all the $k \cdot p$ simulations studying InAs/GaAs CQDs (Refs. 13, 18, and 20-22) have neglected elastic strain, which is known to be important in these structures. In particular, biaxial strain is known to have a major impact on the electronic structure of valence holes in CQDs. ${ }^{26}$ First, it splits $\mathrm{HH}$ and $\mathrm{LH}$ subbands, which should reduce the $\mathrm{HH}-\mathrm{LH}$ mixing. Second, it reverses the confinement potential of LHs, pushing them outside the QDs. ${ }^{27}$ Despite the importance of strain, unstrained calculations are surprisingly consistent with most experimental observations and with atomistic calculations including strain. ${ }^{13}$ In this work we provide an explanation for this discrepancy by considering both strain and the contribution of variable mass parameters.

We study the effect of strain on the formation of antibonding hole ground states in InAs/GaAs CQDs. Holes are described with a four-band $k \cdot p$ Hamiltonian ${ }^{19}$ and biaxial strain is considered within the isotropic elasticity theory. ${ }^{28-30} \mathrm{We}$ show that strain certainly reduces $\mathrm{HH}-\mathrm{LH}$ mixing but also the tunneling of both HHs and LHs. As a result, the interplay between the $\mathrm{HH}$ tunneling rate and the $\mathrm{LH}$ correction holds as in the unstrained case, but the antibonding state is achieved at smaller interdot distances. The inclusion of position-dependent effective masses ${ }^{31}$ further reduces the HH-LH mixing due to the weaker spin-orbit interaction in the GaAs barrier. Thus, the interdot distance at which the 
antibonding state appears is shifted back near the unstrained value. We then proceed to show that the magnetic fieldinduced bonding-to-antibonding transition predicted for GaAs/AlGaAs CQDs (Ref. 23) is, in principle, also feasible in strained InAs/GaAs CQDs. This prediction does not agree with magnetophotoluminescence (PL) measurements that show little change in the energy splitting between bonding and antibonding hole states as a function of increasing longitudinal magnetic fields. We ascribe this disagreement to the traditional implementation of magnetic fields in multiband $k \cdot p$ Hamiltonians, ${ }^{19}$ which exaggerates the HH-LH mixing. Finally we show that alternative implementations of the magnetic field ${ }^{32}$ in the four-band $k \cdot p$ Hamiltonian retrieve the experimental behavior.

\section{THEORY}

Following Refs. 18, 19, and 33, we expand the wave function of the valence hole confined in the nanostructure in terms of $J_{z}=+3 / 2,+1 / 2,-1 / 2,-3 / 2$ Bloch basis functions. In cylindrical coordinates and atomic units, the Hamiltonian reads

$$
\hat{\mathcal{H}}_{\mathrm{LK}}=\left(\begin{array}{cccc}
\hat{P}_{+} & -\hat{S} & \hat{R} & 0 \\
-\hat{S}^{*} & \hat{P}_{-} & 0 & \hat{R} \\
\hat{R}^{*} & 0 & \hat{P}_{-} & \hat{S} \\
0 & \hat{R}^{*} & \hat{S}^{*} & \hat{P}_{+}
\end{array}\right)+\hat{V}^{h} \mathcal{I}+\hat{V}^{\varepsilon}
$$

The operators in the above expression are given by

$$
\begin{gathered}
\hat{P}_{+}=\frac{1}{2}\left[\left(\gamma_{1}+\gamma_{2}\right) \hat{\Pi}_{\perp}^{2}+\left(\gamma_{1}-2 \gamma_{2}\right) \hat{\Pi}_{z}^{2}\right], \\
\hat{P}_{-}=\frac{1}{2}\left[\left(\gamma_{1}-\gamma_{2}\right) \hat{\Pi}_{\perp}^{2}+\left(\gamma_{1}+2 \gamma_{2}\right) \hat{\Pi}_{z}^{2}\right], \\
\hat{R}=\frac{1}{2}(-\sqrt{3}) \gamma_{2} \hat{\Pi}_{-}^{2}, \\
\hat{S}=\frac{1}{2}(2 \sqrt{3}) \gamma_{3} \hat{\Pi}_{-} \hat{\Pi}_{z},
\end{gathered}
$$

where $\gamma_{1,2,3}$ represent the Luttinger material parameters, $\hat{\Pi}_{\perp}^{2}=\hat{\Pi}_{x}^{2}+\hat{\Pi}_{y}^{2}, \hat{\Pi}_{ \pm}=\left(\Pi_{x} \pm i \Pi_{y}\right), \hat{\Pi}_{a}=\hat{p}_{a}-e \mathbf{A}_{a}, \hat{p}_{a}=-i \nabla_{a}$, and $\mathbf{A}=(-y, x, 0) B / 2$, where $B$ is the magnetic field. $\mathcal{I}$ is the identity matrix while $\hat{V}^{h}$ and $\hat{V}^{\varepsilon}$ are the scalar and tensorial potentials induced by quantum confinement and strain, respectively.

For the inclusion of strain, we take the Bir-Pikus Hamiltonian $^{34}$ and then restrict ourselves to the biaxial approximation. ${ }^{35}$ This covers the most relevant effects of strain in (001)-oriented nanostructures. ${ }^{30}$ Within this approximation $\hat{V}^{\varepsilon}$ is diagonal, $\hat{V}^{\varepsilon}(\rho, z)=\left(\hat{P}_{+}^{\varepsilon}, \hat{P}_{-}^{\varepsilon}, \hat{P}_{-}^{\varepsilon}, \hat{P}_{+}^{\varepsilon}\right)$, where:

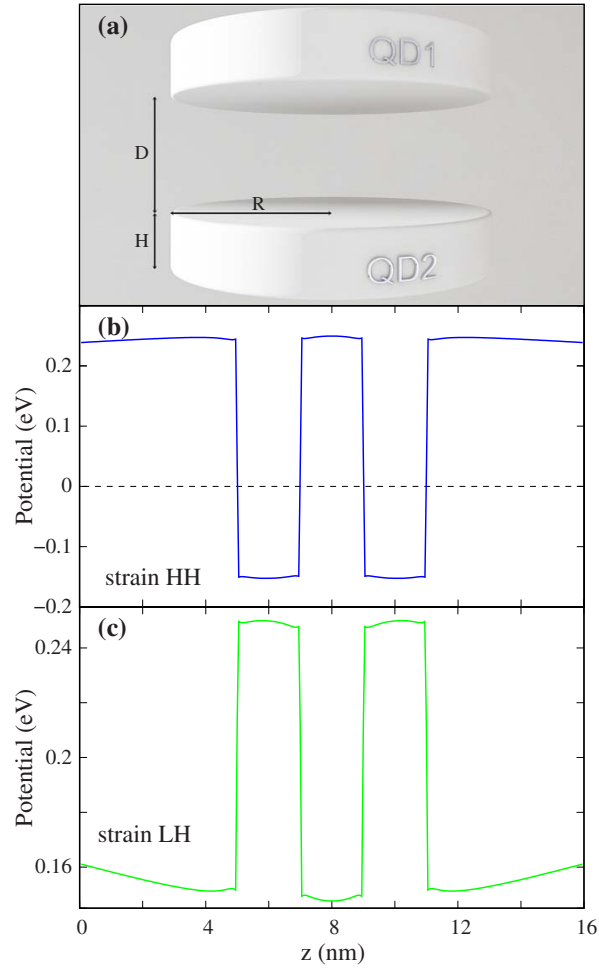

FIG. 1. (Color online) (a) Schematic representation of the CQDs under study. Band-edge profile including biaxial strain (b) for $\mathrm{HH}$ and (c) for $\mathrm{LH}$.

$$
\hat{P}_{+}^{\varepsilon}=\left(2 a_{v}+b\right) \varepsilon_{\perp}+\left(a_{v}-b\right) \varepsilon_{z z}
$$

$$
\hat{P}_{-}^{\varepsilon}=\left(2 a_{v}-b\right) \varepsilon_{\perp}+\left(a_{v}+b\right) \varepsilon_{z z}
$$

Here $a_{v}$ and $b$ are the valence-band deformation potential parameters, $\varepsilon_{i j}$ are the symmetric strain tensor components, and $\varepsilon_{\perp}=\left(\varepsilon_{x x}+\varepsilon_{y y}\right) / 2$, which we calculate within the framework of the isotropic elastic theory. ${ }^{28,29}$ It should be noted that this theory allows for good strain estimates while preserving axial symmetry. ${ }^{30}$

Hamiltonian (1) considers constant Luttinger parameters throughout the entire nanostructure. A more realistic description of hole tunneling should take into account the influence of position-dependent effective masses, i.e., the effect produced by the different Luttinger parameters in the QD and the barrier. To study this, we shall use a four-band BurtForeman Hamiltonian, $\hat{\mathcal{H}}_{B F},{ }^{31,36}$ along with the biaxial strain terms defined above. Since $\hat{\mathcal{H}}_{B F}$ reduces to $\hat{\mathcal{H}}_{\mathrm{LK}}$ in uniform mass systems, a direct comparison of the results of the two Hamiltonians gives insight into the effect of heterogeneous masses.

Both $\hat{\mathcal{H}}_{\mathrm{LK}}$ and $\hat{\mathcal{H}}_{B F}$ exhibit a few symmetries that we use to characterize their eigenstates, known as Luttinger spinors. Because of the circular symmetry of the CQD we study, illustrated in Fig. 1(a), the states can be classified by its total angular momentum $F_{z}=m_{z}+J_{z}$, where $m_{z}$ and $J_{z}$ are the envelope and Bloch angular momenta of the spinor components. Although the Hamiltonian is also invariant under the 
inversion center, $\hat{i}$, reflection $\hat{\sigma}_{x y}$ is not a good quantum number for the entire Luttinger spinor. Individual components of the spinor, however, do have well-defined mirror symmetry and can be classified as symmetric (bonding, $\nu_{z}=b$ ) or antisymmetric (antibonding, $\nu_{z}=a b$ ) with respect to inversion. Because the spinor components can be decomposed as $f_{m_{z}}(\mathbf{r})=f(\rho, z) e^{i m_{z} \theta}$, we can rewrite $\hat{i}=\hat{c}_{2}^{z} \hat{\sigma}_{x y}$, where $\hat{c}_{2}^{z}$ is a binary rotation around the growth direction $z$ and $\hat{\sigma}_{x y}$ is the reflection plane in between the two QDs. Then, $\hat{i} f_{m_{z}}(\mathbf{r})=e^{i m_{z} \pi} \hat{\sigma}_{x y} f_{m_{z}}(\mathbf{r})$. It follows that the spinor has welldefined chirality, i.e., is an eigenfunction of the chirality operator $^{18}$

$$
\hat{\nu}=\left(\begin{array}{cccc}
\hat{\sigma}_{x y} & 0 & 0 & 0 \\
0 & -\hat{\sigma}_{x y} & 0 & 0 \\
0 & 0 & \hat{\sigma}_{x y} & 0 \\
0 & 0 & 0 & -\hat{\sigma}_{x y}
\end{array}\right) .
$$

In CQDs, the two lowest-lying states normally correspond to the Luttinger spinors with $\left|F_{z}, \nu\right\rangle=| \pm 3 / 2, \uparrow\rangle$ and $| \pm 3 / 2, \downarrow\rangle$. For our analysis, it is convinient to write explicitly the symmetries of these states ${ }^{37}$

$$
|3 / 2, \uparrow\rangle=\left(\begin{array}{c}
f_{0, b}(\mathbf{r})\left|J_{z}=+\frac{3}{2}\right\rangle \\
f_{1, a b}(\mathbf{r})\left|J_{z}=+\frac{1}{2}\right\rangle \\
f_{2, b}(\mathbf{r})\left|J_{z}=-\frac{1}{2}\right\rangle \\
f_{3, a b}(\mathbf{r})\left|J_{z}=-\frac{3}{2}\right\rangle
\end{array}\right)
$$

and

$$
|3 / 2, \downarrow\rangle=\left(\begin{array}{c}
f_{0, a b}(\mathbf{r})\left|J_{z}=+\frac{3}{2}\right\rangle \\
f_{1, b}(\mathbf{r})\left|J_{z}=+\frac{1}{2}\right\rangle \\
f_{2, a b}(\mathbf{r})\left|J_{z}=-\frac{1}{2}\right\rangle \\
f_{3, b}(\mathbf{r})\left|J_{z}=-\frac{3}{2}\right\rangle
\end{array}\right) .
$$

In the expressions above $f_{m_{z}, \nu_{-}}(\mathbf{r})$ represent the envelope functions of the spinor and $\left|J_{z}\right\rangle$ the Bloch part. The dominant component in both cases is the $J_{z}=+3 / 2$ one, as it corresponds to a $\mathrm{HH}$ with the lowest angular momentum, $m_{z}=0$. This means that $|3 / 2, \uparrow\rangle$ is essentially a bonding $\mathrm{HH}$ and $|3 / 2, \downarrow\rangle$ an antibonding HH. However, in both cases the second largest component is $J_{z}=+1 / 2$, i.e., a LH with opposite molecular character. This component is responsible for the ground-state reversal at long interdot distances. ${ }^{18}$

Since we are interested in the effect of strain, we maximize it by considering pure InAs CQDs embedded in a pure GaAs matrix. The QDs are modeled as quantum disks [see Fig. 1(a)] of height $H$ and radius $R$, and the inter- dot distance is labeled as $D$. The confining potential is a square well whose depth is given by the band offset, $V=200 \mathrm{meV}$. For InAs, we use Luttinger parameters $\left(\gamma_{1}, \gamma_{2}, \gamma_{3}\right)=(20.0,8.5,9.2),{ }^{38}$ deformation potential constants $a_{v}=0.66 \mathrm{eV}$ and $b=-1.8 \mathrm{eV}$, and lattice constant $a_{0}=6.06 \AA .^{30}$ For GaAs, we use $\left(\gamma_{1}, \gamma_{2}, \gamma_{3}\right)$ $=(6.98,2.06,2.93),{ }^{38}$ deformation potential constants $a_{v}=0.70 \mathrm{eV}$ and $b=-2.0 \mathrm{eV}$, and lattice constant $a_{0}=5.65 \AA .{ }^{30}$ The Poisson coefficient is assumed isotropic, $c=1 / 3$.

The four-band $k \cdot p$ Hamiltonian is integrated using a finite differences scheme and the resulting matrices are diagonalized using the Arnoldi iterative method. ${ }^{39}$

\section{RESULTS AND DISCUSSION}

\section{A. Identical CQDs}

We start by investigating the case of two identical QDs (homonuclear molecule). The QDs have $R=15 \mathrm{~nm}$ and $H=2 \mathrm{~nm}$. As a first approximation we employ InAs Luttinger parameters for the entire structure. The important contribution of position-dependent Luttinger parameters will be discussed below. The spatial confinement potential along the CQD axis is the same for both HH and LH, but the inclusion of the strain contribution introduces significant differences. This is illustrated in Fig. 1 for HH [panel (b)] and for $\mathrm{LH}$ [panel (c)] in a CQD with interdot distance $D=2 \mathrm{~nm}$. For the HH, strain lowers the band edge below the unstrained InAs value (dashed line, zero energy), thus increasing the confinement. By contrast, for the LH the band edge is raised. This introduces a splitting between the $\mathrm{HH}$ and LH minima of about $300 \mathrm{meV}$. In addition, the LH bandedge profile is reversed. The potential inside the QDs becomes repulsive and the LH will tend to localize in the barrier region. ${ }^{26,27}$ It is clear from these effects that strain can induce important changes in the electronic structure of the CQDs, especially when LH plays a role.

To visualize the influence of strain on the electronic structure, we compare the energies of the two lowest hole states, namely, $|3 / 2, \uparrow\rangle$ (hereafter bonding state) and $|3 / 2, \downarrow\rangle$ (hereafter antibonding state). The molecule dissociation spectrum is depicted in Fig. 2, using solid (dashed) lines for the bonding (antibonding) state. Figure 2(a) shows the case without strain. The result is as expected. ${ }^{18}$ Namely, at short interdot distances the ground state is bonding, but it is replaced by the antibonding state after a critical distance of $D_{c}=2.6 \mathrm{~nm}$. Figure 2(b) shows the case with strain. The picture is qualitatively similar, except for the presence of a constant energy stabilization with increasing $D$, which is due to the strain relaxation as the QDs are separated. ${ }^{24}$ The main conclusion is that the bonding-antibonding crossover still takes place, although at shorter interdot distances.

For a better insight of the ground-state reversal, in Fig. 2(c) we plot the tunneling rate $t$, which we define as the energy splitting between bonding and antibonding states. For short interdot distances it is positive (the ground state is bonding) and then it switches to negative (the ground state becomes antibonding). The magnitude of the negative tunneling rate in the presence of strain (thick line) is just as 


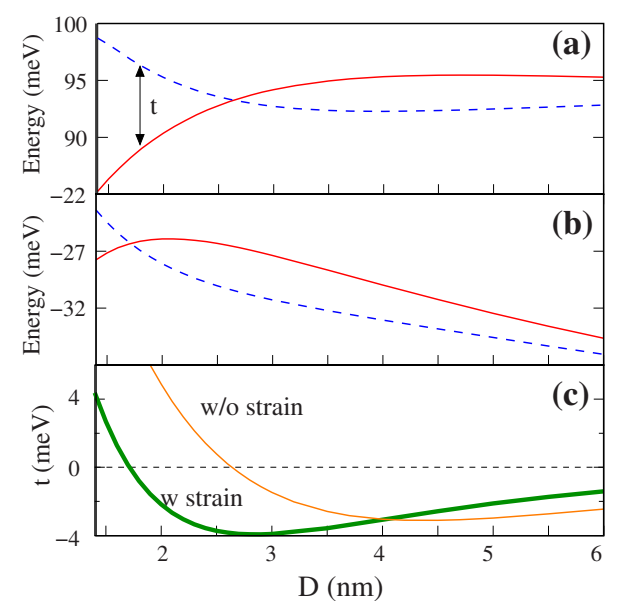

FIG. 2. (Color online) Hole energy as a function of the interdot distance in the (a) absence and (b) presence of strain. Bonding (antibonding) state is represented with solid (dashed) line. (c) Tunneling rate in the strained and unstrained cases.

strong as in its absence (thin line), indicating the antibonding ground state is robust. The decay with $D$ is, however, faster.

For further insight into the impact of strain, in Fig. 3 we study the weight of the LH components in the ground state. In the absence of strain (thin line), the LHs constitute around $\sim 2 \%$ of the spinor weight at short distances, when the ground state is bonding. The $\mathrm{LH}$ contribution increases to about $\sim 4 \%$ at $D=2.7 \mathrm{~nm}$, when the ground state becomes antibonding. ${ }^{18}$ The inclusion of strain (thick line) further reduces these amounts. The largest LH contribution now does not exceed $2 \%$. This is due to the energy splitting between $\mathrm{HH}$ and $\mathrm{LH}$ subbands originated from the different $\mathrm{HH}$ and LH potentials, shown in Fig. 1, which increases the HH character of the low-energy states. Based on this result, it is surprising that the magnitude of the negative tunneling rate [Fig. 2(c)] is barely affected by strain. Using a toy model, in Ref. 18 we suggested that the reduced weight of the LH component could be compensated by an enhancement of its tunneling rate, due to the location of the band-edge minimum in the interdot barrier. Real calculations of strain confirm this minimum [Fig. 1(c)]. To test if this really enhances LH tunneling, in the inset of Fig. 3 we plot the charge density of the

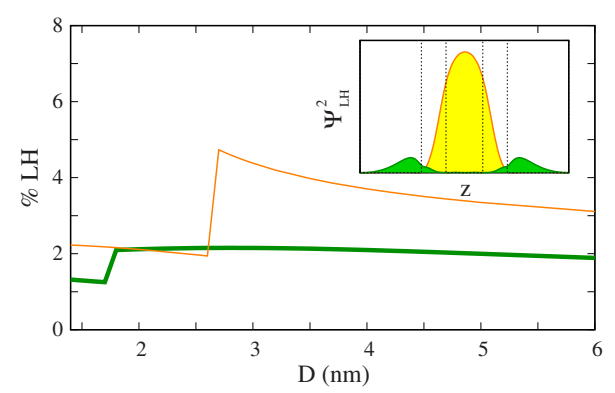

FIG. 3. (Color online) Weight of LH components in the groundstate spinor as a function of the interdot distance. Thick (thin) lines are used for strained (unstrained) CQDs. The inset shows the dominant LH charge density in a CQD with $D=3 \mathrm{~nm}$. Dark green (light orange) areas correspond to strained (unstrained) cases. Dotted lines represent the two QD limits.

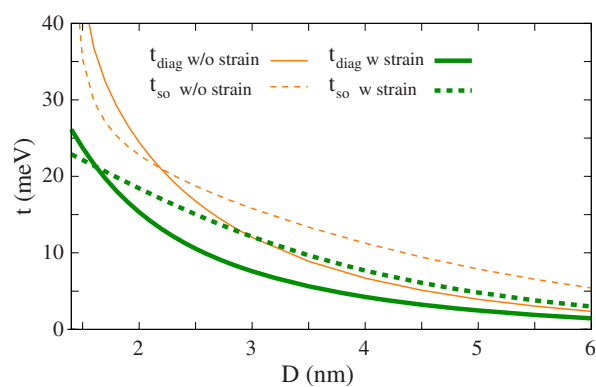

FIG. 4. (Color online) Diagonal tunneling rate (solid lines) and off-diagonal correction (dashed lines) as a function of the interdot distance. Thick (thin) lines are used for strained (unstrained) CQDs.

dominant LH component along the molecular axis in a CQD with $D=3 \mathrm{~nm}$. Dark green (light orange) areas correspond to strained (unstrained) wave functions and dotted lines show the QD limits. Clearly, strain removes charge density from the QDs. However, it does not concentrate the LH in the interdot region, as initially expected. Instead, most of the charge is deposited on the sides of the QDs and the interdot region is severely depleted. It then follows that there is no enhancement of LH tunneling. An alternative explanation of the robust antibonding ground state is thus needed.

The origin of the negative tunneling rates is the offdiagonal correction to the $\mathrm{HH}$ tunneling. ${ }^{18}$ To fully understand the effect of strain it is worth comparing its influence on the diagonal elements and the off-diagonal correction terms. The effective tunneling rate is the energy splitting between the bonding and antibonding states of the LK Hamiltonian

$$
t_{\text {eff }}=E_{|3 / 2, \downarrow\rangle}-E_{|3 / 2, \uparrow\rangle} .
$$

In order to separate contributions in $t_{\text {eff }}$, we split the Hamiltonian into diagonal and off-diagonal parts, $\hat{\mathcal{H}}_{\mathrm{LK}}=\hat{\mathcal{H}}_{\mathrm{LK}}^{\text {diag }}$ $+\hat{\mathcal{H}}_{\mathrm{LK}}^{\text {off }}$. The former describes the diagonal tunneling, which is mostly given by $\mathrm{HH}$ tunneling with a small $\mathrm{LH}$ contribution. The latter contains the terms mixing different spinor components, thus capturing the off-diagonal correction. Note that the off-diagonal correction includes spin-orbit interaction as well as nonrelativistic terms of the $k \cdot p$ expansion. ${ }^{19}$ We can then define the following elements:

$$
\begin{gathered}
t_{\text {diag }}=\left\langle 3 / 2, \downarrow\left|\hat{\mathcal{H}}_{\mathrm{LK}}^{\text {diag }}\right| 3 / 2, \downarrow\right\rangle-\left\langle 3 / 2, \uparrow\left|\hat{\mathcal{H}}_{\mathrm{LK}}^{\text {diag }}\right| 3 / 2, \uparrow\right\rangle, \\
t_{\text {off }}=-\left(\left\langle 3 / 2, \downarrow\left|\hat{\mathcal{H}}_{\mathrm{LK}}^{\text {off }}\right| 3 / 2, \downarrow\right\rangle-\left\langle 3 / 2, \uparrow\left|\hat{\mathcal{H}}_{\mathrm{LK}}^{\text {off }}\right| 3 / 2, \uparrow\right\rangle\right),
\end{gathered}
$$

which fulfill $t_{\text {eff }}=t_{\text {diag }}-t_{\text {off }}$. At this point, we can investigate the effect of strain in each of these contributions. This is shown in Fig. 4. Thick (thin) lines are used for the strained (unstrained) system and solid (dashed) lines are used for $t_{\text {diag }}$ $\left(t_{\text {off }}\right)$. For short interdot distances $t_{\text {diag }}>t_{\text {off }}$ and the ground state is bonding, $|3 / 2, \uparrow\rangle$. However, at longer distances $t_{\text {off }}>t_{\text {diag }}$ and the ground state becomes antibonding, $|3 / 2, \downarrow\rangle$.

Figure 4 confirms that strain reduces the diagonal tunneling. The off-diagonal correction is also reduced but for short interdot distances the reduction is smaller. This is the key result, as it enables the off-diagonal correction to overcome 


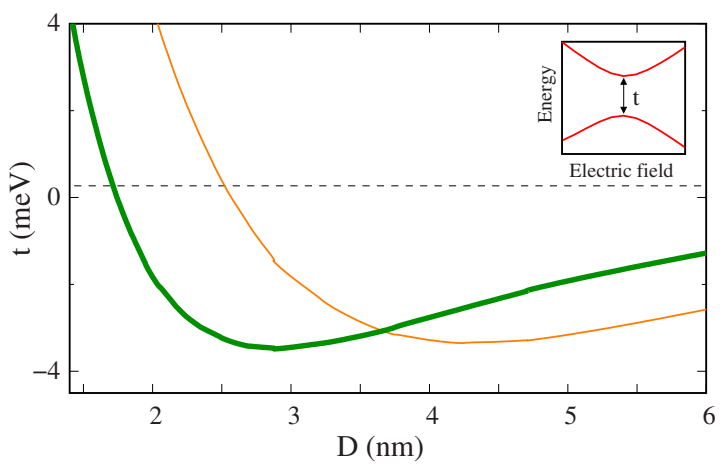

FIG. 5. (Color online) Same as Fig. 2(c) but for an asymmetric CQD subject to resonant electric fields. The inset shows the typical electric field dependence of the lowest hole energy levels.

diagonal coupling at short interdot distances, thus explaining the effect of strain observed in Fig. 2.

\section{B. Nonidentical CQDs}

Real self-assembled CQDs are often asymmetric and so is their strain field. Molecular coupling in these structures is achieved by applying vertical electric fields which tune the energy levels of the individual QDs. ${ }^{4-6,13}$ Next, we study if the role of strain in CQDs made of nonidentical quantum disks differs from that of the homonuclear case. We model a CQD where the upper disk (QD1) has $R=15 \mathrm{~nm}$ and $H=1.8 \mathrm{~nm}$ while the lower disk (QD2) has $R=13 \mathrm{~nm}$ and $H=2.2 \mathrm{~nm}$. In these series of calculations, we keep using homogeneous InAs Luttinger parameters for the entire structure. For each interdot distance $D$, we determine the resonant electric field providing molecular coupling and evaluate the corresponding tunneling rate (see inset in Fig. 5). The resulting tunneling rates are shown in Fig. 5. As can be seen, asymmetric molecules subject to resonant electric fields behave as symmetric molecules. Strain simply lowers the critical distance of the bonding-antibonding reversal and introduces a faster decay of the tunneling with $D$.

\section{Position-dependent mass}

In the previous sections we have used InAs Luttinger parameters for the entire CQD structure. This approximation relies on the fact that most of the charge density is located inside the QDs, and it has been used in all the $k \cdot p$ studies of holes in CQDs so far. ${ }^{13,18,20-24}$ One may argue that, while this approximation is reasonable for single QDs, it may give a deficient description of tunneling through the barrier in CQDs. This is even more likely in strained CQDs, where LHs tend to localize outside the QDs (Fig. 3 inset).

In this section we use a Burt-Foreman Hamiltonian, $\hat{\mathcal{H}}_{B F}$, with InAs Luttinger parameters inside the QDs and GaAs ones in the barrier. We study the tunneling of holes in the symmetric CQD. The molecule-dissociation spectrum is depicted in Fig. 6. Panel (a) shows unstrained CQDs and panel (b) strained CQDs. By comparing Fig. 6(a) with the constant mass spectrum, Fig. 2(a), we see that the main effect of the position-dependent mass is to quench the bonding-

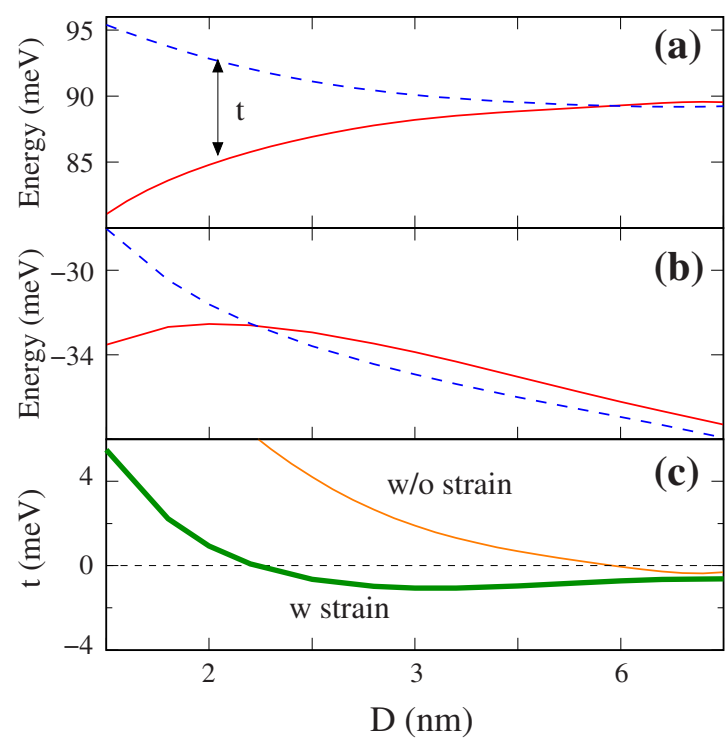

FIG. 6. (Color online) Same as Fig. 2 but using $\hat{\mathcal{H}}_{B F}$ with different Luttinger parameters in the InAs QD and the GaAs barrier.

antibonding reversal, which now occurs at much longer $D$ and is very weak. This is better seen in panel (c), where the tunneling rates are represented. The negative values attained by the tunneling rate are smaller than in Fig. 2(c). The origin of this quenching is the smaller spin-orbit interaction in GaAs $\left(\gamma_{3}=2.93\right)$ as compared to InAs $\left(\gamma_{3}=9.2\right)$, which reduces $t_{\text {off }}$.

The inclusion of strain shifts the distance for the groundstate crossover, $D_{c}$, back to smaller interdot separations, owing to the mechanism described in the Sec. III A. Interestingly, using a complete description of the system one obtains $D_{c}=2.2 \mathrm{~nm}$, which is close to the estimated value using a constant mass and neglecting strain, $D_{c}=2.6 \mathrm{~nm}$. This is because strain and variable mass act in opposite directions.

\section{Magnetic field dependence}

In Ref. 23, we predicted that the bonding-to-antibonding ground-state crossover could be induced after growth using longitudinal magnetic fields. For this to occur, the interdot distance had to be smaller than the critical distance of the crossover. The magnetic field was then used to supply the additional HH-LH coupling strength needed for $t_{\text {off }}$ to overcome $t_{\text {diag. }}$ This prediction was formulated for strain-free systems. In this section we investigate if it persists in the presence of strain. To this end, we first calculate the magnetic field response of the lowest hole states of a symmetric, strained CQD with $D=1.5 \mathrm{~nm}$ (the critical distance for the crossover is $D=1.7 \mathrm{~nm}$ ). For simplicity, the states are calculated using homogeneous masses. The results are shown in Fig. 7(a). One can see that, in spite of the strain, the bondingto-antibonding crossover still occurs.

Next, we compare the theoretical expectation with experimental results on an InAs CQD. We determine the energy splitting between the lowest antibonding and bonding states, $\Delta_{B A B}$, in an InAs CQD. The calculated energy splitting corresponds to the difference between the lowest energy solid 


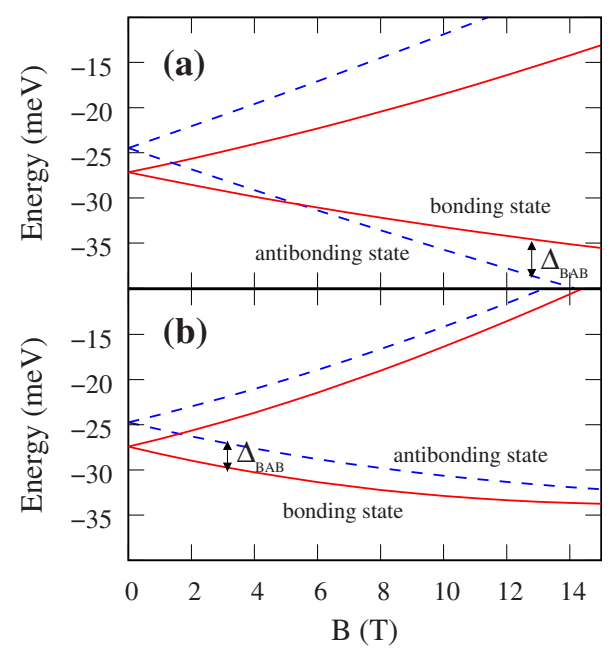

FIG. 7. (Color online) Hole energy levels as a function of a longitudinal magnetic field in a strained CQD with $D=1.5 \mathrm{~nm}$. (a) shows the results using $\hat{\mathcal{H}}_{\mathrm{LK}}$. (b) shows the alternative results using the magnetic terms of $\hat{\mathcal{H}}_{B}$.

and dashed lines displayed in Fig. 7. The experimentally measured energy splitting is obtained from the measured PL spectra of the neutral exciton in a CQD sample with interdot barrier thickness $D=2 \mathrm{~nm}$. In parallel to the calculated case, this thickness is somewhat below the experimentally observed critical thickness for the reversal of bonding and antibonding ground states $(\sim 3 \mathrm{~nm}) .{ }^{13}$ The energies of all four molecular orbital states are measured at the applied electric field where the hole states of the two dots are in resonance, i.e., the electric field at which coherent hole tunneling forms molecular wave functions. A detailed description of the experimental methods can be found in previous publications. ${ }^{9,40}$ The difference in energy between the lowest energy bonding and antibonding states $\left(\Delta_{B A B}\right)$ is directly calculated from these data. ${ }^{41}$

The values of $\Delta_{B A B}$ observed in the experiment and determined by the theoretical model are shown in Fig. 8 for different fields, using symbols and dashed lines, respectively. At $B=0 \mathrm{~T}, \Delta_{B A B}$ is positive (the ground state is bonding) in both theory and experiment. However, one can see that $\Delta_{B A B}$

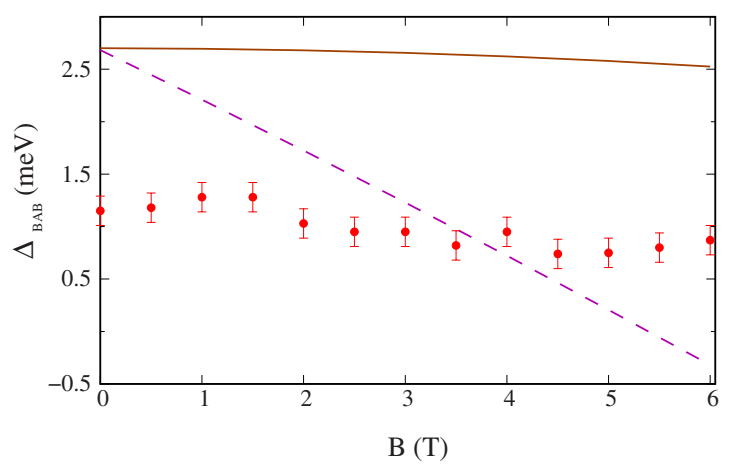

FIG. 8. (Color online) Energy splitting between antibonding and bonding ground states as a function of the magnetic field. Solid line: theoretical estimate using $\mathcal{H}_{B}$. Dashed line: theoretical estimate using $\mathcal{H}_{\mathrm{LK}}$. Symbols: experimental results. in the experiment is roughly constant against the magnetic field. This trend is contrary to that obtained with $\mathcal{H}_{\mathrm{LK}}$, where a strong decrease with $B$ is observed, eventually leading to a change of sign (i.e., bonding-to-antibonding reversal). Using more realistic CQD shapes, inhomogeneuos masses or different tunneling conditions at $B=0 \mathrm{~T}$ does not change the theoretical trend.

At this point, we suggest the discrepancy between theory and experiment arises from $\hat{\mathcal{H}}_{\mathrm{LK}}$ overestimating the magnetic field effect in heterostructures. In the LK formulation, the presence of an external magnetic field can be introduced by means of the following prescription: ${ }^{19,33}$ the differential equation fulfilled by the envelope function is modified by replacing the momentum $p_{\alpha}$ by the canonical momentum $p_{\alpha}-e A_{\alpha}$, where $A$ is the vector potential of the magnetic field. This results in the widely used Hamiltonian (1), which includes magnetic field terms in both diagonal and offdiagonal components of the four-band Hamiltonian. An alternative formulation was proposed by some of us in Ref. 32, in which the momentum $p_{\alpha}$ is also replaced by the canonical momentum $p_{\alpha}-e A_{\alpha}$, but in the original Hamiltonian. Then, the envelope function approximation is applied, i.e., the Hamiltonian acts on a wave function written as a sum of products of envelope and Bloch functions and, after left multiplying by proper Bloch functions, integration is carried out over the unit cell, thus yielding a set of coupled differential equations for the envelope components. As a result, the fourband Hamiltonian contains magnetic terms in the diagonal only. This formulation is more consistent with the envelope function approximation and provided a more regular behavior for holes confined in quantum ring structures. ${ }^{42}$

We next recalculate the magnetic field spectrum of the CQD using Ref. 32 approximation. The magnetic terms now read

$$
\mathcal{H}_{B}=\left(\begin{array}{cccc}
B_{3 / 2} & 0 & 0 & 0 \\
0 & B_{1 / 2} & 0 & 0 \\
0 & 0 & B_{-1 / 2} & 0 \\
0 & 0 & 0 & B_{-3 / 2}
\end{array}\right),
$$

where

$$
\begin{aligned}
& B_{3 / 2}=\left(\gamma_{1}+\gamma_{2}\right)\left[\frac{B^{2} \rho^{2}}{8}+\frac{\left(F_{z}-1\right) B}{2}\right], \\
& B_{1 / 2}=\left(\gamma_{1}-\gamma_{2}\right)\left[\frac{B^{2} \rho^{2}}{8}+\frac{\left(F_{z}-1 / 3\right) B}{2}\right], \\
& B_{-1 / 2}=\left(\gamma_{1}-\gamma_{2}\right)\left[\frac{B^{2} \rho^{2}}{8}+\frac{\left(F_{z}+1 / 3\right) B}{2}\right], \\
& B_{-3 / 2}=\left(\gamma_{1}+\gamma_{2}\right)\left[\frac{B^{2} \rho^{2}}{8}+\frac{\left(F_{z}+1\right) B}{2}\right] .
\end{aligned}
$$

The mass coefficients we have used are those which correctly retrieve the single-band limit in case of band decoupling. ${ }^{43}$ The magnetic field dependence of the CQD spectrum using Eq. (10) is illustrated in Fig. 7(b). Clearly, 
the ground state is now bonding for all fields. What is more, if we plot $\Delta_{B A B}$ obtained with $\mathcal{H}_{B}$ as a function of the magnetic field, solid line in Fig. 8, we note that the splitting is roughly constant, in agreement with the experimental trend. This behavior is quite robust to changes in the tunneling conditions at $B=0 \mathrm{~T}$, as it essentially depends on the magnetic terms.

To close this section, we note that the value of $\Delta_{B A B}$ estimated with $\mathcal{H}_{B}$ is slightly larger than the experimental value because we model an ideal CQD and omit positiondependent mass effects. Also, the magnetic field-induced splittings between $F_{z}= \pm 3 / 2$ states in Fig. 7 are significantly larger than that typically observed in experiments. ${ }^{11}$ The large splitting originates in the finite orbital linear magnetic term of the dominant HH component in Eqs. (11a) and (11d). The inclusion of further refinements in the model may make up for these quantitative disagreements. Nonetheless, the results in this section reveal that off-diagonal magnetic terms lead to qualitative disagreement with experimental trends and Ref. 32 offers a plausible justification for their absence.

\section{SUMMARY}

We have shown that the formation of antibonding hole ground states in InAs/GaAs CQDs (Refs. 13 and 18) is robust against the inclusion of both strain and positiondependent Luttinger parameters in the theory. Biaxial strain increases the $\mathrm{HH}$ character of the Luttinger spinors and reduces the tunneling of both $\mathrm{HH}$ and $\mathrm{LH}$. The correction to the tunneling rate induced by $\mathrm{HH}-\mathrm{LH}$ mixing is also reduced, but to a smaller extent. As a result, the bonding-toantibonding reversal occurs at shorter interdot distances than in unstrained CQDs. The inclusion of strain components beyond the biaxial approximation used in this work are known to lead to slight variations of the potential near the QD interfaces. ${ }^{30}$ These additional effects are secondary when compared to biaxial strain, and they are likely to be blurred in real CQDs by compositional grading and interface irregularities.

The inclusion of position-dependent Luttinger parameters further decreases the strength of the HH-LH mixing due to the weak spin-orbit interaction (and hence weak HH-LH mixing) in the GaAs barrier. This shifts the distance of the ground-state reversal back to larger interdot distances. The competition between the effects of strain and positiondependent effective masses explains the good agreement between sophisticated atomistic calculations and simpler $k \cdot p$ models neglecting strain and variable mass. ${ }^{13}$

We further investigated the feasibility of inducing the bonding-to-antibonding transition with magnetic fields in strained InAs/GaAs CQDs, as previously predicted for (unstrained) GaAs/AlGaAs CQDs. ${ }^{23}$ This would be of interest for flexible after-growth control of the optoelectronic properties of the nanostructure. Calculations using a LK implementation of the magnetic field indicate that the transition is possible. However, no trace of such effect is observed in magneto-PL experiments, where the splitting between bonding and antibonding states remains nearly constant with the field. We have shown that the origin of this disagreement can be ascribed to the traditional formulation of the magnetic field terms in the LK Hamiltonian, which artificially enhances the HH-LH mixing. Using the alternative implementation proposed in Ref. 32, the experimental behavior is retrieved.

\section{ACKNOWLEDGMENTS}

Support from MCINN under Project No. CTQ200803344, UJI-Bancaixa under Project No. P1-1A2009-03, and the Ramon y Cajal program (J.I.C.) is acknowledged. *josep.planelles@qfa.uji.es; http://quimicaquantica.uji.es/

${ }^{1}$ W. G. van der Wiel, S. De Franceschi, J. M. Elzerman, T. Fujisawa, S. Tarucha, and L. P. Kouwenhoven, Rev. Mod. Phys. 75, 1 (2002).

${ }^{2}$ M. Scheibner, A. S. Bracker, D. Kim, and D. Gammon, Solid State Commun. 149, 1427 (2009).

${ }^{3}$ M. Bayer, P. Hawrylak, K. Hinzer, S. Fafard, M. Korkusiński, Z. R. Wasilewski, O. Stern, and A. Forchel, Science 291, 451 (2001).

${ }^{4}$ H. J. Krenner, M. Sabathil, E. C. Clark, A. Kress, D. Schuh, M. Bichler, G. Abstreiter, and J. J. Finley, Phys. Rev. Lett. 94, 057402 (2005).

${ }^{5}$ A. S. Bracker, M. Schneiber, M. F. Doty, E. A. Stinaff, I. V. Ponomarev, J. C. Kim, L. J. Whitman, T. L. Reinecke, and D. Gammon, Appl. Phys. Lett. 89, 233110 (2006).

${ }^{6}$ G. Ortner, M. Bayer, Y. Lyanda-Geller, T. L. Reinecke, A. Kress, J. P. Reithmaier, and A. Forchel, Phys. Rev. Lett. 94, 157401 (2005).

${ }^{7}$ G. Burkard, G. Seelig, and D. Loss, Phys. Rev. B 62, 2581 (2000).
${ }^{8}$ E. A. Stinaff, M. Schneibner, A. S. Bracker, I. V. Ponomarev, V. L. Korenev, M. E. Ware, M. F. Doty, T. L. Reinecke, and D. Gammon, Science 311, 636 (2006).

${ }^{9}$ M. F. Doty, J. I. Climente, A. Greilich, M. Yakes, A. S. Bracker, and D. Gammon, Phys. Rev. B 81, 035308 (2010).

${ }^{10}$ H. Y. Ramirez and S. J. Cheng, Phys. Rev. Lett. 104, 206402 (2010).

${ }^{11}$ M. F. Doty, M. Scheibner, I. V. Ponomarev, E. A. Stinaff, A. S. Bracker, V. L. Korenev, T. L. Reinecke, and D. Gammon, Phys. Rev. Lett. 97, 197202 (2006).

${ }^{12}$ S. Tomic, T. S. Jones, and N. M. Harrison, Appl. Phys. Lett. 93, 263105 (2008)

${ }^{13}$ M. F. Doty, J. I. Climente, M. Korkusiński, M. Scheibner, A. S. Bracker, P. Hawrylak, and D. Gammon, Phys. Rev. Lett. 102, 047401 (2009).

${ }^{14}$ W. Jaskólski, M. Zieliński, and G. W. Bryant, Acta Phys. Pol. A 106, 193 (2004).

${ }^{15}$ W. Jaskólski, M. Zieliński, G. W. Bryant, and J. Aizpurua, Phys. Rev. B 74, 195339 (2006).

${ }^{16}$ G. Bester, J. Shumway, and A. Zunger, Phys. Rev. Lett. 93, 
047401 (2004); G. Bester, A. Zunger, and J. Shumway, Phys. Rev. B 71, 075325 (2005).

${ }^{17}$ M. Korkusiński, W. Sheng, P. Hawrylak, Z. Wazilewski, G. Ortner, M. Bayer, A. Babinski, and M. Potemski, AIP Conf. Proc. 772, 685 (2005).

${ }^{18}$ J. I. Climente, M. Korkusiński, G. Goldoni, and P. Hawrylak, Phys. Rev. B 78, 115323 (2008).

${ }^{19}$ J. M. Luttinger and W. Kohn, Phys. Rev. 97, 869 (1955).

${ }^{20}$ C. Y. Hsieh, R. Cheriton, M. Korkusiński, and P. Hawrylak, Phys. Rev. B 80, 235320 (2009).

${ }^{21}$ J. I. Climente, M. Korkusinski, G. Goldoni, and P. Hawrylak, Physica E (Amsterdam) 40, 1862 (2008).

${ }^{22}$ T. Chwiej and B. Szafran, Phys. Rev. B 81, 075302 (2010).

${ }^{23}$ J. I. Climente, Appl. Phys. Lett. 93, 223109 (2008).

${ }^{24}$ A. I. Yakimov, A. A. Bloshkin, and A. V. Dvurechenskii, Semicond. Sci. Technol. 24, 095002 (2009).

${ }^{25}$ F. Michelini, M. A. Dupertuis, S. Dalessi, and E. Kapon, (unpublished).

${ }^{26}$ C. Pryor, Phys. Rev. Lett. 80, 3579 (1998).

${ }^{27}$ W. Sheng and J. P. Leburton, Phys. Status Solidi B 237, 394 (2003).

${ }^{28}$ J. R. Downes, D. A. Faux, and E. P. O’Reilly, J. Appl. Phys. 81, 6700 (1997).

${ }^{29}$ J. H. Davies, J. Appl. Phys. 84, 1358 (1998).

${ }^{30}$ M. Tadic, F. M. Peeters, K. L. Janssens, M. Korkusiński, and P. Hawrylak, J. Appl. Phys. 92, 5819 (2002).

${ }^{31}$ B. A. Foreman, Phys. Rev. B 48, 4964 (1993).

${ }^{32}$ J. Planelles and W. Jaskólski, J. Phys.: Condens. Matter 15, L67 (2003).

${ }^{33}$ M. Pacheco and Z. Barticevic, J. Phys.: Condens. Matter 11,
1079 (1999).

${ }^{34}$ G. L. Bir and G. E. Pikus, Symmetry and Strain-Induced Effects in Semiconductors (Wiley, New York, 1974).

${ }^{35}$ Calvin Yi-Ping Chao and S. L. Chuang, Phys. Rev. B 46, 4110 (1992).

${ }^{36}$ L. C. Lew Yan Voon and M. Willatzen, The $k \cdot p$ Method, (Springer, New York, 2009), Sec. 12.5.4.

${ }^{37}$ For simplicity, only positive $F_{z}$ instances are analyzed. Analogous reasoning could be followed for their negative counterparts.

${ }^{38}$ I. Vurgaftman, J. R. Meyer, and L. R. Ram-Mohan, J. Appl. Phys. 89, 5815 (2001).

${ }^{39} \mathrm{http}: / / w w w . c a a m . r i c e . e d u / s o f t w a r e / A R P A C K /$

${ }^{40}$ M. F. Doty, M. Scheibner, A. S. Bracker, I. V. Ponomarev, T. L. Reinecke, and D. Gammon, Phys. Rev. B 78, 115316 (2008).

${ }^{41}$ The resonant change in $g$ factor, which is used to identify the orbital character of molecular states (Ref. 13) is neglected in the present theory. For the sample with $2 \mathrm{~nm}$ barrier presented here, the molecular excited state has antibonding character throughout the measured range of applied magnetic fields. Because the antibonding molecular excited state has a larger $g$ factor on resonance, subtracting this effect from the experimental data could only increase the $\Delta_{B A B}$ splitting as a function of increasing magnetic field. Consequently, including the $g$ factor in the theory would not improve the agreement between the measured data and the $\mathcal{H}_{\mathrm{LK}}$ estimate.

${ }^{42}$ J. I. Climente, J. Planelles, and W. Jaskólski, Phys. Rev. B 68, 075307 (2003).

${ }^{43}$ A. S. Andreev, S. P. Andreev, and Yu. A. Gurvich, Laser Phys. 7, 976 (1997). 